\title{
DIFFERENTIALS IN CERTAIN CLASSES OF GRAPHS
}

\author{
P. ROUSHINI LEELY PUSHPAM AND D. YOKESH
}

\begin{abstract}
Let $X \subset V$ be a set of vertices in a graph $G=(V, E)$. The boundary $B(X)$ of $X$ is defined to be the set of vertices in $V-X$ dominated by vertices in $X$, that is, $B(X)=(V-X) \cap N(X)$. The differential $\partial(X)$ of $X$ equals the value $\partial(X)=|B(X)|-|X|$. The differential of a graph $G$ is defined as $\partial(G)=$ $\max \{\partial(X) \mid X \subset V\}$. It is easy to see that for any graph $G$ having vertices of maximum degree $\Delta(G), \partial(G) \geq \Delta(G)-1$. In this paper we characterize the classes of unicyclic graphs, split graphs, grid graphs, $k$-regular graphs, for $k \leq 4$, and bipartite graphs for which $\partial(G)=\Delta(G)-1$. We also determine the value of $\partial(T)$ for any complete binary tree $T$.
\end{abstract}

\section{Introduction}

Let $G=(V, E)$ be a graph. For graph theoretic terminology not given here, refer to Harary [2]. For a vertex $v \in V$, the open neighborhood of $v$ is the set $N(v)=\{u \in V \mid u v \in$ $E\}$ and the closed neighborhood is the set $N[v]=N(v) \cup\{v\}$. For a set $S \subseteq V$, its open neighborhood is $N(S)=\cup_{v \in S} N(u)$ and the closed neighborhood is $N[S]=N(S) \cup S$.

The boundary $B(S)$ of a set $S$ is defined to be the set of vertices in $V-S$ dominated by vertices in $S$, that is $B(X)=(V-S) \cap N(S)$. The differential $\partial(S)$ of $S$ equals the value $\partial(S)=|B(S)|-|S|$. The differential of a graph of $G$ is defined as $\partial(G)=$ $\max \{\partial(S) \mid S \subset V\}$. As reported in [4], the differential of a set was first defined by Hedetniemi [3], and later studied by Mashburn et al. [4] and Goddard and Henning [1]. The minimum differential of an independent set was also studied by Zhang [6].

It is easy to see that for any graph $G$ having vertices of maximum degree $\Delta(G)$, $\partial(G) \geq \Delta(G)-1$. We say that a graph $G$ is a $(\Delta-1)$-differential graph if $\partial(G)=\Delta(G)-1$. In this paper we characterize the classes of unicyclic graphs, split graphs, grid graphs, $k$-regular graphs, for $k \leq 4$, and bipartite graphs that are $(\Delta-1)$-differential. We also determine the value of $\partial(T)$ for any complete binary tree $T$.

Corresponding author: P. Roushini Leely Pushpam.

Received February 27, 2008; revised June 25, 2009.

Key words and phrases. Boundary of a set of vertices, differential of a graph. 


\section{Properties of $(\Delta-1)$-differential graphs}

In this section we determine several properties of $(\Delta-1)$-differential graphs that can be used to characterize the five classes of $(\Delta-1)$-differential graphs in the next section.

Theorem 1. Let $G=(V, E)$ be a connected $(\Delta-1)$-differential graph, let $v \in V$ be a vertex of maximum degree, that is, $|N(v)|=\Delta(G)$, and let $H$ be the subgraph of $G$ induced by $V-N[v]$. Then the following properties hold:

(i) Each connected component of $H$ is either a $K_{1}$ or a $K_{2}$.

(ii) Each vertex $u \in N(v)$ has at most two neighbors in $H$.

(iii) If $S$ is the set of all vertices in $N(v)$ having two neighbors in $H$, and no two members of $S$ have a common neighbor in $H$, then $|S| \leq \Delta-2$.

(iv) The diameter of $G$ is at most 6 .

Proof. Since the theorem clearly holds when $\Delta(G)=1$, we assume that $\Delta(G) \geq 2$.

(i) Let $v$ be a vertex of maximum degree, i.e., $|N(v)|=\Delta(G)$, and assume that $D=\{v\}$ is a $\partial$-set. Let $x$ be a vertex in $H$, the subgraph of $G$ induced by the vertices in $V-N[v]$. If $x$ has more than one neighbor in $H$ then $\partial(D \cup\{x\})>\partial(D)$, contradicting our assumption that $D$ is a $\partial$-set. Hence, each component of $H$ has maximum degree at most 1 , and therefore each component $H$ is either a $K_{1}$ or a $K_{2}$.

(ii) If any vertex $u \in N(v)$ has three or more neighbors in $H$ then $\partial(D \cup\{u\})>\partial(D)$, again contradicting our assumption that $D$ is a $\partial$-set.

(iii) Let $S \subseteq N(v)$ be the set of neighbors of $v$, each of which has two neighbors in $H$ and no two vertices in $S$ have a common neighbor in $H$. In this case, if $|S|=\Delta(G)$ then $\partial(S)>\partial(D)$, again contradicting our assumption that $D$ is a $\partial$-set.

(iv) Since $G$ is connected, and since by (a) each connected component of $H$ is either a $K_{1}$ or a $K_{2}$, it follows that every vertex in $H$ is within distance at most 2 to a vertex in $N(v)$, and therefore is within distance at most 3 to the vertex $v$ of maximum degree $\Delta(G)$. Therefore, every pair of vertices in $G$ are within distance 6 of each other and $\operatorname{diam}(G) \leq 6$.

\section{Characterizations of five classes of $(\Delta-1)$-differential graphs}

In this section we characterize the classes of unicyclic graphs, split graphs, grid graphs, $k$-regular graphs, for $k \leq 4$, and bipartite graphs that are $(\Delta-1)$-differential graphs.

\subsection{Unicylic graphs}

Definition 2. A graph $G$ with $|V|=n$ is a unicyclic graph if it is connected and $|E|=n$; or equivalently, if it is connected and contains exactly one cycle. 
In this section we provide a characterization of the unicyclic graphs that are $(\Delta-1)$ differential graphs. In order to do this we need the family $\mathcal{T}$ of rooted trees and theorem as defined and proved by Mashburn et al. [4].

For a rooted tree $T$, let $T_{u}$ denote the subtree of $T$ rooted at vertex $u$. Let $P_{n}$ denote the path on $n$ vertices.

The family $\mathcal{T}$ consists of all trees $T$ rooted at a vertex $v$ of maximum degree having the following properties:

(i) each vertex $u \in N(v)$ has $\operatorname{deg}(u) \leq 3$,

(ii) the connected components of $T-N[v]$ are $K_{1}$ 's and $K_{2}$ 's, and

(iii) either $N(v)$ contains at least two vertices $u$ for which $T_{u} \in\left\{K_{1}, K_{2}\right\}$ or $N(v)$ contains exactly one leaf and no vertex of $N(v)$ has degree three.

Theorem 3.([4]) A tree $T$ is a $(\Delta-1)$-differential tree if and only if $T \in \mathcal{T}$.

Theorem 4. A unicyclic graph $G$ with cycle $C_{n}$ is a $(\Delta-1)$-differential graph if and only if

(i) $3 \leq n \leq 5$, and

(ii) there exists an edge $e \in C_{n}$ and a vertex of maximum degree in $G$ that is not incident to e such that $G-e \in \mathcal{T}$.

Proof. Let $G$ be a $(\Delta-1)$-differential unicyclic graph having a vertex $v \in V$ of degree $\Delta(G)$. If $v \in V\left(C_{n}\right)$, then by Theorem $1(\mathrm{i}), 3 \leq n \leq 5$. If $v \notin V\left(C_{n}\right)$ we claim that $n=3$. By Theorem 1(ii), each component of the subgraph $H$ induced by the vertices in $V-N[v]$ is a $K_{1}$ or a $K_{2}$. Hence, $v$ is adjacent to at least one vertex of $C_{n}$ and $n=3$. Since $G$ is a $(\Delta-1)$-differential unicyclic graph, $D=\{v\}$ is a $\partial$-set. Let $e$ be an edge in $C_{n}$ such that there is a vertex of maximum degree not incident to $e$. Then $G-e$ is a tree and $\partial(G-e)=(\Delta-1)$. Hence, by Theorem $3, G-e \in \mathcal{T}$.

Conversely, let $G$ satisfy the given conditions. Let $e$ be an edge in $C_{n}$ such that $G-e \in \mathcal{T}$, where there is a vertex $v$ of maximum degree not incident to $e$. Since $G-e \in \mathcal{T}$, by Theorem $3, \partial(G-e)=\Delta(G-e)-1$ and $D=\{v\}$ is a $\partial$-set of $G-e$. Hence, by the choice of $e$ the differential of $G$ does not increase. Therefore, $G$ is a $(\Delta-1)$-differential graph.

\subsection{Split graphs}

In this section we provide a characterization of the class of $(\Delta-1)$-differential split graphs.

Definition 5. A graph $G=(V, E)$ is called a split graph if the vertex set has a bipartition $V=V_{1} \cup V_{2}$, where $V_{1}$ is an independent set (no two vertices in $V_{1}$ are adjacent) and the subgraph $G\left[V_{2}\right]$ induced by $V_{2}$ is a complete graph (every pair of vertices are adjacent). 
Theorem 6. A split graph $G$ with bipartition $\left(V_{1}, V_{2}\right)$ is a $(\Delta-1)$-differential graph if and only if there exist $v \in V_{2}$ with maximum degree $\Delta(G)$ such that the following conditions hold.

(i) Each $u \in V_{2}-\{v\}$ has at most two neighbours in $V_{1}-N(v)$.

(ii) If $S$ is the subset of $V_{2}-\{v\}$ such that each vertex in $S$ has exactly two neighbours in $V_{1}-N(v)$ and no two members of $S$ have a common neighbour in $V_{1}-N(v)$ then $S$ does not dominate $N(v)-\{x\}, x \in V_{1}$.

Proof. Let $G$ be a $(\Delta-1)$-differential split graph having a vertex $v \in V_{2}$ of degree $\Delta(G)$.

Since the theorem clearly holds when $\Delta(G)=1$, we assume that $\Delta(G) \geq 2$.

Suppose there exist a vertex $u \in V_{2}-\{v\}$ which has more than two neighbours in $V_{1}-N(v)$, then $\partial\{u, v\} \geq \Delta-1+3-2=\Delta>\Delta(G)-1=\partial(G)$, which is a contradiction. Hence each vertex in $V_{2}-\{v\}$ has at most two neighbours in $V_{1}-N(v)$. Let $S$ be a subset of $V_{2}-\{v\}$ satisfying condition (ii). Suppose $S$ dominates $N(v)-\{x\}$, then $\partial(S)=2|S|+\Delta-1-|S|-|S|+1=\Delta>\Delta(G)-1=\partial(G)$, which is a contradiction. Hence condition (ii) holds.

Conversely let $G$ satisfy the given conditions. Now $\partial(G) \geq \partial(\{v\})=\Delta(G)-1$. Now to prove $\partial(G) \leq \Delta(G)-1$. Let $D$ be a $\partial$-set, assume $v \in D$. Clearly adding another vertex to $D$ does not increase the differential of $G$. Hence $D=\{v\}$ and $\partial(G)=\Delta(G)-1$. If $v \notin D$, adding a vertex of $V_{1}$ to $D$ does not increase the differential of $G$.

If $D=V_{2}-\{v\}$, then

$\partial(G) \leq \partial(D) \leq 2\left(\left|V_{2}\right|-1\right)+\Delta-\left(\left|V_{2}\right|-1\right)-2-\left(\left|V_{2}\right|-1\right) \leq \Delta-2 \leq \Delta-1$.

Hence $\partial(G)=\Delta(G)-1$.

\subsection{Grid graphs}

In this section we determine all $(\Delta-1)$-differential grid graphs.

Definition 7. The Cartesian product $G \square H$ of a graph $G=(V, E)$ and a graph $H=$ $(W, F)$ is the graph with vertex set equal to the Cartesian product $V \square W$ and two vertices $(u, v)$ and $(w, x)$ are adjacent in the Cartesian product graph if and only if either $u=w$ and $v$ is adjacent to $x$ in $H$, or $u$ is adjacent to $w$ in $G$ and $v=x$.

Definition 8. The $m$ by $n$ grid graph $G_{m, n}$ is the Cartesian product of two paths $P_{m}$ and $P_{n}, G_{m, n}=P_{m} \square P_{n}$.

Theorem 9. $G_{m, n}$ is a $(\Delta-1)$-differential graph if and only if $2 \leq m, n \leq 3$.

Proof. The proof follows directly from Theorem 1(i).

3.4. $k$-regular graphs, for $k \leq 4$

Definition 10. A graph $G=(V, E)$ is called $k$-regular if every vertex $v \in V$ has degree $k$, that is $|N(v)|=k$. 
In this section we study an important property of a regular graph $G$, which is $(\Delta-$ $1)$-differential, that can be used to characterize $k$-regular graphs, which are $(\Delta-1)$ differential, when $1 \leq k \leq 4$.

Theorem 11. A $k$-regular graph $G$ is a $(\Delta-1)$-differential graph, then $k+1 \leq p \leq k+3$ where $p$ is the number of vertices in the graph.

Proof. Let $G$ be a $(\Delta-1)$-differential $k$-regular graph. Let $v \in V$ and $H=V-N[v]$. We claim that $H$ has at most two vertices.

Suppose not, then there exist at least 3 vertices $x, y, z$ in $H$. In view of Theorem $1(\mathrm{i})$ following cases arise.

(i) $\{x, y, z\}$ is independent.

(ii) $x y \in E(G)$ and $y z \notin E(G)$.

(iii) $x y \in E(G)$ and $y z \in E(G)$.

In case (i), $N(x)=N(y)=N(z)=N(v)$ which implies that each vertex in $N(v)$ has three neighbors in $H$, which is a contradiction.

In case (ii), both $x$ and $y$ are adjacent to $k-1$ vertices in $N(v)$. By Theorem 1(ii), each vertex in $N(v)$ has at most two neighbors in $H$. Hence $\operatorname{deg}(z)<k$, which is a contradiction.

Case (iii) can be similarly dealt with. Hence $H$ has at most two vertices. Therefore $k+1 \leq p \leq k+3$.

Theorem 12. A k-regular graph $G$ where $k=1,2$ is a $(\Delta-1)$-differential graph if and only if $G$ is isomorphic to $K_{2}$ or $C_{n}, 3 \leq n \leq 5$.

Proof. If $G$ is isomorphic to either $K_{2}$ or $C_{n}, 3 \leq n \leq 5$ then clearly $\partial(G)=\Delta(G)-1$. Conversely, let $G$ be a $(\Delta-1)$-differential graph, which is $k$-regular, $k=1,2$. If $G$ is 1 -regular, then clearly $G$ is isomorphic to $K_{2}$. Suppose $G$ is 2-regular, then by Theorem 1(i), $G$ is isomorphic to $C_{n}, 3 \leq n \leq 5$.

Theorem 13. A 3-regular graph $G$ is a $(\Delta-1)$-differential graph if and only if $G$ is isomorphic to $K_{4}$ or $K_{3,3}$ or $C_{3} \times K_{2}$.

Proof. Let $G$ be a $(\Delta-1)$-differential 3-regular graph. By Theorem $11,4 \leq p \leq 6$, where $p$ is the number of vertices in $G$. Since the number of vertices of odd degree in any graph is even, $p=4$ or $p=6$. Let $v \in V$ and $v_{i}, i=1,2,3$ be the neighbors of $v$. When $p=4$, clearly $G$ is isomorphic to $K_{4}$.

When $p=6$, let $x, y \in V-N(v)$. If $x, y$ are not adjacent then clearly $N(x)=N(y)$ and $N(v)$ is independent. Therefore $G$ is isomorphic to $K_{3,3}$.

If $x$ and $y$ are adjacent then exactly one member say $v_{1}$ of $N(v)$ is adjacent to both $x$ and $y$, and the other two members $v_{2}, v_{3}$ of $N(v)$ are adjacent to $x$ and $y$ respectively. Further $v_{2}$ and $v_{3}$ are adjacent. Hence $G$ is isomorphic to $C_{3} \times K_{2}$.

Conversely suppose $G$ is isomorphic to $K_{4}$ or $K_{3,3}$ or $C_{3} \times K_{2}$ then clearly $\partial(G)=$ $\Delta(G)-1$. 


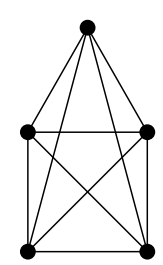

$G_{1}$

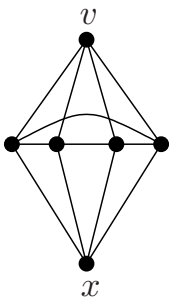

$G_{2}$

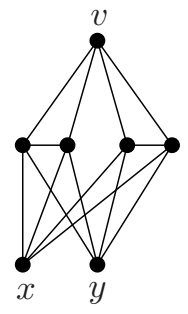

$G_{3}$

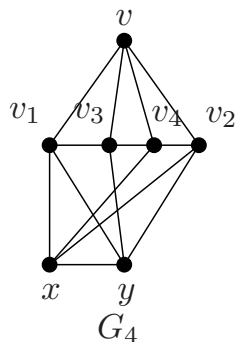

Figure 1:

Theorem 14. A 4-regular graph is a $(\Delta-1)$-differential graph if and only if $G$ is isomorphic to $G_{i}, 1 \leq i \leq 4$ as given in Figure 1 .

Proof. Let $G$ be a $(\Delta-1)$-differential 4-regular graph. By Theorem $11,5 \leq p \leq 7$. When $p=5, G$ is isomorphic to $K_{5}$.

When $p=6$, let $x \in V-N(v)$ where $v \in V$. Let $N(v)=\left\{v_{1}, v_{2}, v_{3}, v_{4}\right\}$. Clearly $x$ is adjacent to each $v_{i}, 1 \leq i \leq 4$ and the subgraph induced by $\left\{v_{1}, v_{2}, v_{3}, v_{4}\right\}$ is a cycle of length 4 . Hence $G$ is isomorphic to $G_{2}$.

When $p=7$, let $x, y \in V-N(v)$. If $x$ and $y$ are not adjacent, then $x$ and $y$ are adjacent to each $v_{i}$ and the subgraph induced by $\left\{v_{1}, v_{2}, v_{3}, v_{4}\right\}$ is $2 k_{2}$. Therefore $G$ is isomorphic to $G_{3}$. If $x$ and $y$ are adjacent then exactly two members of $N(v)$ say $v_{1}$ and $v_{2}$ are adjacent to $x$ and $y$, and the other two members $v_{3}$ and $v_{4}$ of $N(v)$ are such that $x$ and $y$ are adjacent to $v_{3}$ and $v_{4}$ respectively. Further the subgraph induced by $\left\{v_{1}, v_{2}, v_{3}, v_{4}\right\}$ is a $P_{4}$, such that $v_{1}$ and $v_{2}$ are the ends of $P_{4}$. Hence $G$ is isomorphic to $G_{4}$.

Conversely, suppose $G$ is isomorphic to $G_{i}, 1 \leq i \leq 4$, then clearly $G$ is a $(\Delta-1)$ differential graph.

\section{Bipartite graphs}

Definition 15. A graph $G=(V, E)$ is called bipartite if the vertex set can be partitioned into two independent sets.

Finally we provide a characterization of the class of $(\Delta-1)$-differential bipartite graphs. For this purpose we prove the following lemma.

Lemma 16. A bipartite graph with bipartition $(X, Y)$ is a $(\Delta-1)$-differential graphs, then the following holds.

(i) Every vertex in $N(v)$ is of degree at most 3 and every vertex in $Y-N(v)$ is of degree 1.

(ii) Every vertex in $X-\{v\}$ has at most one neighbor in $Y-N(v)$. 


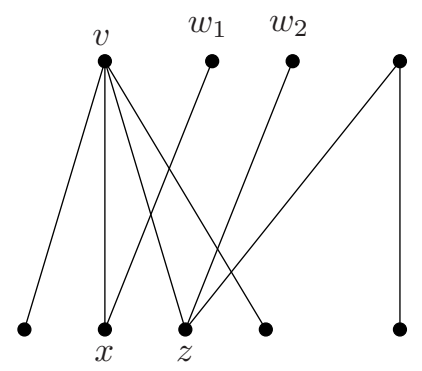

Figure 2:

Proof. Let $v$ be a vertex of maximum degree $\Delta(G)$. If there exist a vertex $y \in N(v)$ of degree more than three, there $\partial(\{v, y\}) \geq \Delta(G)+3-2-1=\Delta(G) \geq \Delta(G)-1=\partial(G)$, which is a contradiction. Hence every member in $N(v)$ is of degree at most three.

If there exists a vertex $w \in Y-N(v)$ of degree more than 1 , then $\partial\{v, w\} \geq \Delta+2-2=$ $\Delta(G) \geq \Delta(G)-1=\partial(G)$, which is a contradiction. Hence every vertex in $Y-N(v)$ is of degree 1 .

If there exists a vertex $x \in X-\{u\}$ which has more than one neighbor in $Y-N(v)$ then $\partial\{x, v\} \geq \Delta+2-2=\Delta(G)>\Delta(G)-1=\partial(G)$, which is a contradiction. Hence each vertex in $X-\{v\}$ has at most one neighbor in $Y-N(v)$.

Definition 17. Let $G$ be a bipartite graph with bipartition $(X, Y)$. Let $v$ be a vertex in $X$ of maximum degree $\Delta(G)$. A vertex $x \in N(v)$ with $\operatorname{deg}(x)=3$ and $N(x)-\{v\}=$ $\left\{w_{1}, w_{2}\right\}$ is said to be a special vertex if either $w_{1}$ or $w_{2}$ has a neighbor $z$ in $N(v)$, then $z$ has a neighbor in $X-\left\{v, w_{1}, w_{2}\right\}$ which is a support. In Figure 2, $x$ is a special vertex.

Theorem 18. A bipartite graph with bipartition $(X, Y)$ is a $(\Delta-1)$-differential graph if and only if there exist a vertex $v$ say in $X$ of maximum degree $\Delta(G)$ such that the following conditions hold:

(i) Every vertex in $N(v)$ is of degree at most three and every vertex in $Y-N(v)$ is of degree one.

(ii) Every vertex in $X-\{v\}$ has almost one neighbor in $Y-N(v)$.

(iii) If $S_{1}$ is the set of all vertices in $N(v)$ which are adjacent to a support in $X-\{v\}$ and $S_{2}$ is the set of all vertices in $N(v)-S_{1}$ which are of degree three, such that no two members of $S_{2}$ have a common neighbor in $X-\{v\}$, then

$$
\text { when } S_{2}=\phi,\left|S_{1}\right| \leq \begin{cases}\Delta-2 & \text { if there exists a special vertex in } S_{1} \\ \Delta-1 & \text { otherwise }\end{cases}
$$

Further when $\left|S_{1}\right|=\Delta-1, v$ is a support. When $S_{2} \neq \phi$, then $\left|S_{1}\right|+\left|S_{2}\right| \leq \Delta(G)-2$.

Proof. Let $G$ be a $(\Delta-1)$-differential bipartite graph. Let $v$ be a vertex of maximum degree $\Delta(G)$. By Lemma 16, conditions (i) and (ii) hold. 
Let $S_{1}$ be the set of all vertices in $N(v)$ which are adjacent to a support $X-\{v\}$ and $S_{2}$ be the set of all vertices in $N(v)-S_{1}$ which are of degree three, such that no two members of $S_{2}$ have a common neighbor in $X-\{v\}$. Let $A$ be the set of vertices in $X-\{v\}$ which are supports. If $S_{2}=\phi$ and there exists a special vertex in $S_{1}$, then we claim $\left|S_{1}\right| \leq \Delta-2$. Suppose not, then $\partial(A)=|A|+\left|S_{1}\right|+1-|A|=\left|S_{1}\right|+1 \geq$ $\Delta(G)-1+1=\Delta(G)>\Delta(G)-1=\partial(G)$, which is a contradiction.

If $S_{2}=\phi$ and if there exists no special vertex in $S_{1}$, then we claim $\left|S_{1}\right| \leq \Delta-1$. Suppose not, then $\partial(A)=|A|+\left|S_{1}\right|-|A|=\left|S_{1}\right| \geq \Delta(G)$, which is a contradiction.

If $\left|S_{1}\right|=\Delta-1$, then we claim that $v$ is a support. Suppose not, then $\partial(\{A, w\})=$ $|A|+\left|S_{1}\right|+2-|A|-1=\left|S_{1}\right|+1=\Delta$, where $w$ is the vertex of degree one adjacent to $v$. Hence $\partial(\{A, w\})>\partial(G)$, which is a contradiction.

If $S_{2} \neq \phi$, then we claim $\left|S_{1}\right|+\left|S_{2}\right| \leq \Delta-2$. Suppose not, then

$$
\begin{aligned}
\partial\left(A \cup S_{2}\right) & =|A|+\left|S_{1}\right|+2\left|S_{2}\right|+1-|A|-\left|S_{2}\right| \\
& =\left|S_{1}\right|+\left|S_{2}\right|+1 \\
& \geq \Delta-1+1 \\
& =\Delta \geq \Delta(G)-1=\partial(G)
\end{aligned}
$$

which is a contradiction. Hence $\left|S_{1}\right|+\left|S_{2}\right| \leq \partial(G)$.

Conversely if $G$ satisfy the given conditions then $\partial(G) \geq \partial(\{v\})=\Delta(G)-1$. To show that $\partial(G) \leq \Delta(G)-1$, let $D$ be a $\partial$-set. Assume first that $v \in D$. Since conditions (i) and (ii) hold, adding another vertex to $D$ does not increase the differential of $G$. Hence we assume that $D=\{v\}$ and $\partial(G)=\Delta(G)-1$. Suppose $v \notin D$. Let $S_{1}$ and $S_{2}$ be as given in the theorem. Let $A$ be the set of all vertices in $X-\{v\}$ which are supports. Let $S_{2}=\phi$. If there exist a special vertex in $S_{1}$, then there exist at least two vertices $x, y$ such that $x, y \notin S_{1}$. Now $\partial(G) \leq \partial(A)=\left|S_{1}\right|+|A|+1-|A|=\left|S_{1}\right|+1 \leq \Delta-1$. Further adding $x$ or $y$ to $A$ does not increase the differential of $G$. Suppose there does not exist a special vertex in $S_{1}$, then $\partial(G) \leq \partial(A)=\left|S_{1}\right|+|A|-|A| \leq \Delta-1$. Let $S_{2} \neq \phi$, then $\partial(G) \leq \partial\left(A \cup S_{2}\right)=\left|S_{1}\right|+|A|+2\left|S_{2}\right|+1-|A|-\left|S_{2}\right|=\left|S_{1}\right|+\left|S_{2}\right|+1 \leq \Delta-2+1=\Delta-1$. Hence $\partial(G) \leq \Delta(G)-1$.

\section{The differential $\partial(T)$ of a complete binary tree $T$}

Finally we calculate the value of $\partial(G)$ for a complete binary tree.

Theorem 19. Let $G$ be a complete binary the consisting of $k$ levels, then

$$
\partial(G)=\left\{\begin{array}{ll}
3 \sum_{i=0}^{\left[\frac{k-1}{3}\right]} \frac{2^{k-2}}{8^{i}} & k=3 n \text { or } 3 n+2 \\
3 \sum_{i=0}^{\left[\frac{k-1}{3}\right]-1} \frac{2^{k-2}}{8^{i}}+1 & k=3 n+1
\end{array} .\right.
$$


Proof. Let $G$ be a complete binary tree. Let $S_{i}$ be the set of all vertices in level $i$ and $\left|S_{i}\right|=n_{i}$, then $n_{i}=2^{i}$.

Since $n_{k}>n_{k-1}>n_{k-2}>\cdots>n_{0}$, clearly $S_{k-1} \cup S_{k-4} \cup \cdots \cup S_{0}$ is a $\partial$-set if $k=3 n$. If $k=3 n$,

$$
\begin{aligned}
\partial(G) & =\left[\left(2^{k}+2^{k-2}+2^{k-3}+2^{k-5}+\cdots\right)-\left(2^{k-1}+2^{k-4}+2^{k-7}+\cdots\right)\right]-1 \\
& =\left[2^{3 n}\left(1+\frac{1}{2^{2}}+\frac{1}{2^{3}}+\frac{1}{2^{5}}+\cdots\right)-2^{3 n}\left(\frac{1}{2}+\frac{1}{2^{4}}+\frac{1}{2^{7}}+\cdots\right)\right]-1 \\
& =\left[2^{3 n}\left(1+\frac{1}{2}+\frac{1}{2^{2}}+\frac{1}{2^{3}}+\cdots\right)-2^{3 n} \frac{2}{2}\left(\frac{1}{2^{3}}+\frac{1}{2^{6}}+\frac{1}{2^{9}}+\cdots\right)\right]-1 \\
& =8^{n}\left[\frac{2 \cdot 8^{n}-1}{8^{n}}-\frac{8}{7}\left(\frac{8^{n}-1}{8^{n}}\right)\right]-1=\frac{6}{7}\left(8^{n}-1\right)
\end{aligned}
$$

Also $\partial(G)=3 \sum_{i=0}^{\left[\frac{3 n-1}{3}\right]} \frac{2^{3 n-2}}{8^{i}}=3 \sum_{i=0}^{n-1} \frac{2^{3 n-2}}{8^{i}}$

$$
\begin{aligned}
& =\frac{38^{n}}{4}\left[1+\frac{1}{8}+\frac{1}{8^{2}}+\cdots+\frac{1}{8^{n-1}}\right] \\
& =\frac{6}{7}\left(8^{n}-1\right)
\end{aligned}
$$

If $k=3 n+1$,

$$
\begin{aligned}
\partial(G) & =\left[2^{3 n+1}+2^{3 n-1}+2^{3 n-2}+\cdots\right]-\left[2^{3 n}+2^{3 n-3}+2^{3 n-6}+\cdots\right] \\
& =2^{3 n}\left[2+1+\frac{1}{2}+\frac{1}{2^{2}}+\cdots\right]-2(8)^{n}\left[1+\frac{1}{2^{3}}+\frac{1}{2^{6}}+\cdots\right] \\
& =\frac{12\left(8^{n}\right)-5}{7}
\end{aligned}
$$

Also $\partial(G)=\left[3 \sum_{i=0}^{\left[\frac{k-1}{3}\right]-1} \frac{2^{k-2}}{8^{i}}\right]+1$

$$
\begin{aligned}
& =\frac{3\left(8^{n}\right)}{2} \times \frac{8}{7}\left(\frac{8^{n}-1}{8^{n}}\right)+1 \\
& =\frac{12\left(8^{n}\right)-5}{7}
\end{aligned}
$$

If $k=3 n+2$,

$$
\partial(G)=\left(2^{3 n+2}+2^{3 n}+2^{3 n-1}+2^{3 n-3}+\cdots+2^{0}\right)
$$




$$
\begin{aligned}
& -\left(2^{3 n+1}+2^{3 n-2}+2^{3 n-5}+\cdots\right) \\
= & \left(2^{0}+2^{1}+2^{2}+\cdots+2^{3 n+2}\right)-2 \times 2\left(1+2^{3}+2^{6}+\cdots\right) \\
= & \left(\frac{2^{3 n+3}-1}{2-1}\right)-4\left(\frac{\left(2^{3}\right)^{n+1}-1}{2^{3}-1}\right) \\
= & \frac{3}{7}\left(8(8)^{n}-1\right)
\end{aligned}
$$

Also $\partial(G)=\left[3 \sum_{i=0}^{\left[\frac{k-1}{3}\right]} \frac{2^{3 n}}{8^{i}}\right]$

$$
=3\left(8^{n}\right)\left[1+\frac{1}{8}+\frac{1}{8^{2}}+\cdots+\frac{1}{8^{n}}\right]=\frac{3}{7}\left(8(8)^{n}-1\right) \text {. }
$$

Hence the theorem is proved.

\section{References}

[1] W. Goddard and M. A. Henning, Generalized domination and independence in graphs, Congr. Numer., 123:161-171, 1997.

[2] F. Harary, Graph Theory, Addison Wesley, Reading Mass., 1972.

[3] S. T. Hedetniemi, Private communication.

[4] J. L. Mashburn, T. W. Haynes, S. M. Hedetniemi, S. T. Hedetniemi and P. J. Slater, Differentials in graphs, Utilitas Math, 69(2006), 43-54.

[5] A. McRae and D. Parks, Private communication.

[6] C. Q. Zhang, Finding critical independent sets and critical vertex subsets are polynomial problems, SIAM J. Discrete Math., 3 (1990),k 431-438.

Department of Mathematics, D.B. Jain College, Chennai 600 097, Tamil Nadu, India.

E-mail: roushinip@yahoo.com

Department of Mathematics, SMK Fomra Institute of Technology, Chennai 603 103, Tamil Nadu, India. 